\title{
Rare case of monarthritis of the knee indicative of tabetic arthropathy
}

\author{
Amghar J*, Benhammou M, Aharram S, Daoudi A and Agoumi O \\ Orthopaedic Trauma Service A, Mohammed VI University Hospital, Faculty of Medicne and Pharmacy, Mohammed I University, Oujda. Morocco
}

\begin{abstract}
With the current sparcity of syphilitic manifestations, the once obvious aspects of the affection have been forgotten or are little known. Tabetic arthropathy, destructive neurogenic condition, due to loss of painful and proprioceptive innervation. It has become exceptional thanks to the early treatment of syphilis with penicillin. We report the case of a 58 year old patient, consultant for a knee arthritis revealing a tabetic arthropathy.
\end{abstract}

\section{Introduction}

Tabs is a syphilitic nerve disease characterized by damage to the spinal cord, which manifests itself as deep tenderness, damage to certain cranial nerves and disorders of the spinal cord. trophic [1]. Joint damage, or arthrotabetes, is a chronic condition that affects one or more joints, deprived of their painful and proprioceptive sensory innervation and continually subjected to the traumas of everyday life. Tabular arthropathy is a typical example of so-called "nervous" arthropathy. It occurs in $10 \%$ of cases of tabs, and has become exceptional thanks to early treatment with penicillin [2]. The often little-known early forms mimic a common gonarthrosis, and our observation illustrates this unusual form.

\section{Observation}

This is a 58-year-old patient who consulted for monoarthritis of the right knee, which has been progressing for 6 months without fever, associated with a biological inflammatory syndrome. The joint puncture brought back a sterile inflammatory fluid.

Clinical examination noted varus knees, with a painless deformity and patellar shock in the left knee (Figure 1). The flexion was less than $60^{\circ}$, with great internal and external ligament laxity. There was sensory neuropathy, with abolished osteotendinous reflexes.

Radiographs of the knee from the front and side showed major osteo-cartilaginous joint destruction destruction of the medial femoral condyle with osteolysis of the medial border of the medial tibial plateau associated with multiple bone constructions with the presence of large intra-articular fragments (Figure 2).

The pathological samples were not specific (Figure 3).

The diagnosis of tabular arthropathy in its hypertrophic form was made on the basis of a 20-year history of syphilitic inoculation canker, posterior radiculocordal syndrome, imaging data, and positive TPHA -VDRL (Treponema Pallidum Hemagglutination Assay-Venereal Disease Research Laboratory) syphilitic serology in blood and CSF. HBV, HCV and HIV serologies were negative.

Penicillin G treatment was given, with regular serological checks until negativation. In view of the extent of joint destruction and

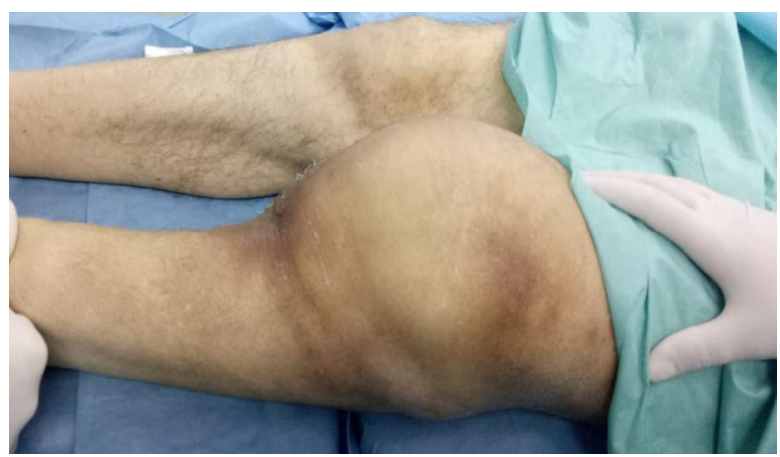

Figure 1. Left knee deformity

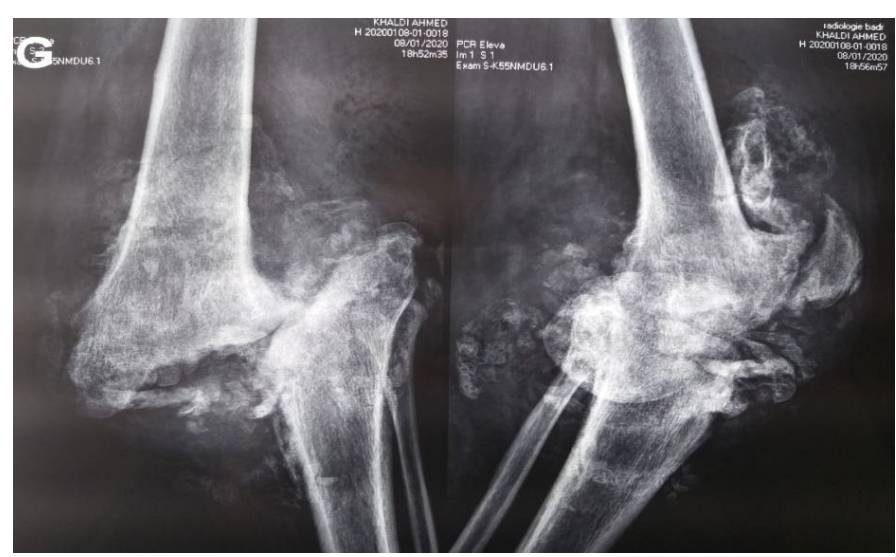

Figure 2. Radiograph of the knee face and profile: destruction of the medial tibial plateau, loss of contact between the articular surfaces and calcifications of the soft tissues

${ }^{\star}$ Correspondence to: Amghar Jawad, Traumatology-Orthopaedics A Department, Mohammed VI University Hospital, Faculty of Medicine and Pharmacy of Oujda, Oujda, Morocco, Tel: 00212626931158; E-mail: jawadamghar2003@gmail.com

Key words: arthritis, knee, tabetic arthropathy, syphilis, joint destruction

Received: March 20, 2020; Accepted: March 29, 2020 Published: April 05, 2020 


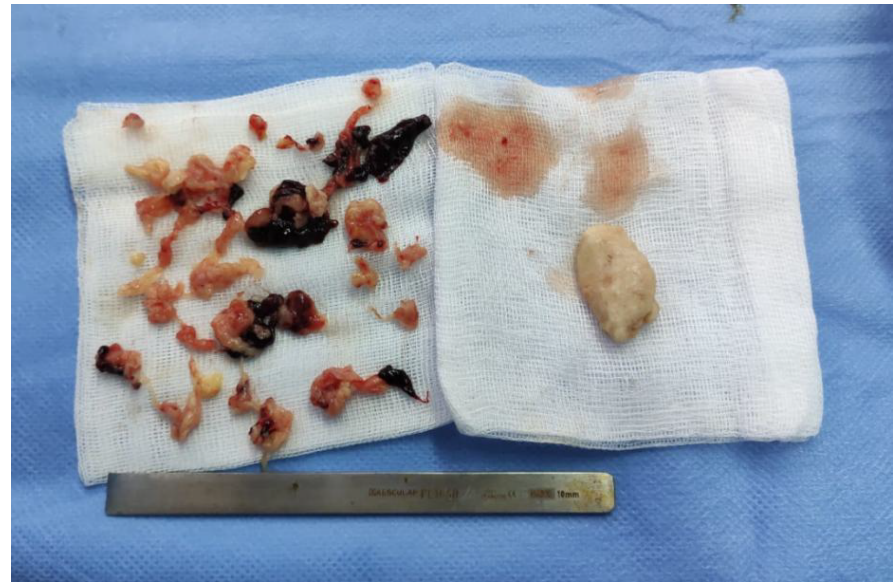

Figure 3. Anatomo-pathological samples from the knee joint with the presence of foreign bodies

the major risk of sepsis on the material, there was no indication for prosthetic arthroplasty. Given the risk, a trans-femoral amputation was performed.

\section{Discussion}

Arthrotabetes is a dreaded complication of neurosyphilis [3], which has become rare due not only to the reduced frequency of the disease, but also to its earlier and better managed treatment. It often occurs 15 to 30 years after the initial syphilitic infection [4]. In $5 \%$ to $10 \%$ of cases, it may precede the clinical signs of tertiary syphilis.

Both sexes are usually affected with the same frequency [5]. The lower limbs are predominantly affected (60 to 75\%), with the knee, ankle, ankle, tarsus, hip, shoulder and elbow being affected in decreasing order of frequency. Involvement is usually mono or pauci-articular, but polyarticular forms affecting up to eight joints have been described. Involvement may be bilateral, symmetrical, and usually rhizomelic.

The time to onset of arthropathies during the course of tabulation varies, as illustrated by a study of 132 cases in which joint damage occurred [6]:

- 21 times during the prodromal period of the table

- 38 times between the first and fifth year

- 32 times between the fifth and tenth year

- 41 times after the tenth year

The onset of tabetic arthropathy is sudden, with a cracking sensation and a loosening of the legs. The joint swelling is sudden and reaches its maximum after a few hours or a few days. It often extends to the entire limb. The characteristic feature of these arthropathies is indolence, contrasting with the extent of joint effusion and destruction, as is the case in this patient $[6,7]$.
Nevertheless, more painful tabetic arthropathy occur when the small joints (hand, foot) are affected. Complications may occur during the course of the disease:

- Spontaneous fractures of the diaphysis or a joint extremity have been described. The appearance of the joint may be altered in the case of intra-articular fractures. This is the case in this patient (Figure 1).

- suppurations following imprudent puncture or during general illness (pneumonia) have also been described.

The diagnosis of bone syphilis is serological: VDRL, TPHA, FTA (Fluorescent Treponemal Antibody). H. Hooshmand et al. [8] reported the results of a series in which the latter test was positive in all patients. In neurosyphilis, parenteral penicillin $\mathrm{G}$ is the most effective treatment, with the administration of 4 million intravenous penicillin $G$ units every 4 hours for $10-15$ days $[9,10]$. The course of tabetic arthropathy may be characterized by fusion of destroyed bone pieces with complete ankylosis. The essential treatment is rest and joint immobilization (removal of support, splints, bed rest). Taken early, arthropathy can heal and the joint can return to its normal architecture. At a later stage, only prosthesis or arthrodesis is useful to improve the disability. However, prostheses should be avoided on this weight-bearing joint because of the hyperlaxity of the tendon structures, which would compromise its stability.

\section{Conclusion}

Tabetic arthropathy is a rare entity at present. It is still possible in some countries such as Morocco, but its diagnosis must be made in the face of any destructive and painless joint damage. The best treatment remains the prevention of syphilis, a sexually transmitted disease.

\section{References}

1. El Fatimi A, Mkinsi O, Etaouil N, Ben Yahya E, Janani S, et al. (1997) Tabetes arthropathies of the knee: 44 observations. Rhumatol 49: 22-26.

2. WHO (1995) Global prevalence and incidence of selected curable sexually transmitted diseases: overview and estimates. World Health Organization, Geneva.

3. Hsaini Y, Mounach J, Satté A, Zerhouni A, Qacif H (2007) Arthrotabes: about five cases. Rev Méd Int 28: 101.

4. Bontoux D (1968) Nervous arthropathies. Cah Coll Med 9: 18-26.

5. El Maghraoui K, Janani S, Yacoubi A, Faiz S, Benyahya E (2005) Tabetes arthropathies of the knee (about 8 cases). Rev Maroc Chir Orthop Traumato 23: 25-2.

6. Marie P (1892) Tabetes arthropathies. Weekly medical and surgical gazette. Medical Wednesdays 29

7. El Maghraoui K, Janani S, Yacoubi A (2005) Tabetes arthropathies of the knee (About 8 cases). Revue Marocaine de Chirurgie Orthopédique et Traumatologique 23: 25-27.

8. Hooshmand H, Escobar MR, Kopf SW (1972) Neurosyphilis a study of 241 patients. JAMA 219: 726-729. [Crossref]

9. Ducas J, Robson HG (1981) Cerebrospinal fluid penicillin levels during therapy for latent syphilis. JAMA 246: 2583-2584. [Crossref]

10. Workowski KA (1998) guidelines for treatment of sexually transmitted diseases Centres for disease control and prevention. MMWR Recomm Rep 47: 1-111. [Crossref]

Copyright: (C2020 Amghar J. This is an open-access article distributed under the terms of the Creative Commons Attribution License, which permits unrestricted use, distribution, and reproduction in any medium, provided the original author and source are credited. 